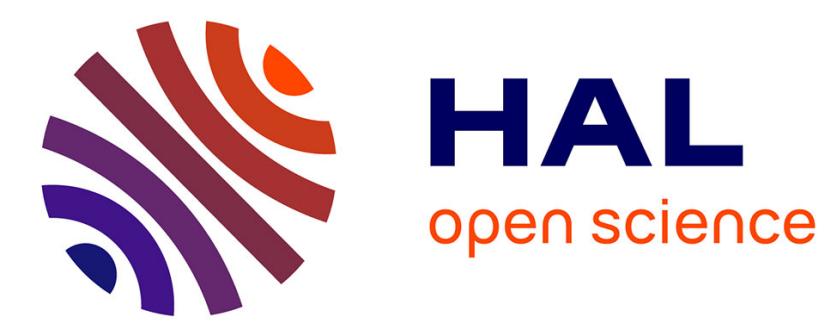

\title{
Non-steady-state photo-EMF in semi-insulating GaAs under frequency-modulated illumination
}

\author{
M Bryushinin, V Kulikov, I Sokolov, Philippe Delaye, Gilles Pauliat
}

\section{To cite this version:}

M Bryushinin, V Kulikov, I Sokolov, Philippe Delaye, Gilles Pauliat. Non-steady-state photo-EMF in semi-insulating GaAs under frequency-modulated illumination. EPL - Europhysics Letters, 2014, 105, pp.64003. 10.1209/0295-5075/105/64003 . hal-01192669

\section{HAL Id: hal-01192669 \\ https://hal-iogs.archives-ouvertes.fr/hal-01192669}

Submitted on 3 Sep 2015

HAL is a multi-disciplinary open access archive for the deposit and dissemination of scientific research documents, whether they are published or not. The documents may come from teaching and research institutions in France or abroad, or from public or private research centers.
L'archive ouverte pluridisciplinaire HAL, est destinée au dépôt et à la diffusion de documents scientifiques de niveau recherche, publiés ou non, émanant des établissements d'enseignement et de recherche français ou étrangers, des laboratoires publics ou privés. 


\title{
Non-steady-state photo-EMF in semi-insulating GaAs under frequency-modulated illumination
}

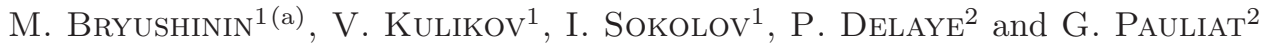 \\ 1 Ioffe Physical Technical Institute - Politekhnicheskaya 26, 194021, St. Petersburg, Russia \\ 2 Laboratoire Charles Fabry, Institut d'Optique, CNRS, Univ Paris-Sud-2 av. A. Fresnel, \\ 91127 Palaiseau cedex, France
}

received 16 January 2014; accepted in final form 5 March 2014

published online 26 March 2014

PACS 42.65.Sf - Dynamics of nonlinear optical systems; optical instabilities, optical chaos and
complexity, and optical spatio-temporal dynamics

PACS $42.70 . \mathrm{Nq}$ - Other nonlinear optical materials; photorefractive and semiconductor materials

\begin{abstract}
We report the excitation of the non-steady-state photoelectromotive force using a uniformly accelerated motion of the recording light pattern. Such illumination is created by the linear frequency modulation of the interfering light beams. The pulsed response is observed experimentally in a semi-insulating GaAs crystal at a light wavelength of $633 \mathrm{~nm}$. The evolution of the pulse shape vs. sweep rate is demonstrated. We also discuss the possible application of the effect in laser Doppler velocimeters and accelerometers.
\end{abstract}

Copyright (C) EPLA, 2014

Introduction. - Since its discovery in 1986, the effect of the non-steady-state photo-EMF [1] has found a lot of applications as a tool for material characterization and a method for detection of phase-modulated optical signals. The effect reveals itself as an alternating current arising in a semiconductor illuminated by an oscillating interference pattern [1-3]. The related techniques realized with an interference pattern running with constant velocity are called holographic current [4] or moving photocarrier grating technique [5]. The electric current appears due to spatial shifts of the space-charge and photoconductivity distributions in the crystal volume. The utilization of a light pattern running with constant velocity usually results in a dc output signal, while the techniques using an oscillating pattern provide an ac one.

The excitation of the photo-EMF signal involves processes of carrier generation, diffusion and drift in an electric field, recombination to local centers. This complex nature of the effect makes it a powerful tool for the determination of photoelectric parameters such as the type and value of photoconductivity, carrier lifetime, mobility and diffusion length, density of recombination centers, etc. $[3,5-8]$.

The running or oscillating interference patterns are usually created by two coherent light beams shifted by frequency or phase modulated by a sinusoidal signal,

\footnotetext{
(a) E-mail: mb@mail.ioffe.ru
}

respectively. A constant frequency shift can arise because of the Doppler effect, while the sinusoidal phase modulation appears in the beam reflected by a vibrating object. Corresponding schemes of velocimeters and vibrometers using photoconductive materials have been proposed [9-13].

In this study we try to extend the variety of the photoEMF techniques introducing the linear frequency modulation (LFM) in the light beams forming an interference pattern. The pattern turns out to be uniformly accelerated in this case. One can expect that the linear frequency modulation will result in a pulsed output signal. Indeed, the response of the highly resistive material to initial fast variations of the light intensity is negligibly small. At the moment, when the interference pattern slows down and stops, a noticeable space-charge grating is recorded, and the signal can appear. Then the pattern begins to run fast again and the signal vanishes. In the following sections we shall consider this effect in detail and discuss its possible applications.

Experimental arrangements. - The experimental setup used for measurements of the non-steady-state photo-EMF signal is shown in fig. 1. The light from the He-Ne laser $\left(\lambda=633 \mathrm{~nm}, P_{\text {out }} \simeq 30 \mathrm{~mW}\right)$ passes through two acousto-optic modulators ML-201-1, which introduce the frequency shifts $f_{0}$ and $f_{0}+\Delta f(t)$ into diffracted light beams $\left(f_{0}=80 \mathrm{MHz}\right)$. We use the output of the 


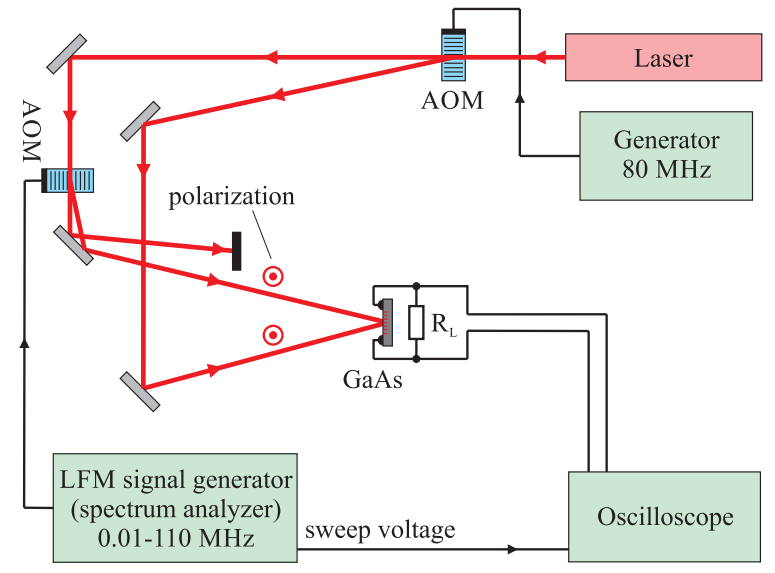

Fig. 1: (Colour on-line) Experimental setup used for measurements of the non-steady-state photo-EMF signal excited by frequency-modulated light. AOM are the acousto-optic modulators.

spectrum analyzer SK4-59 as a generator of the linearfrequency-modulated signal because of its wide range of frequency deviation. Then these beams are directed to the sample forming the interference pattern with average intensity $I_{0}=240 \mathrm{~mW} / \mathrm{cm}^{2}$, contrast $m=0.97$ and spatial frequency $K=190 \mathrm{~mm}^{-1}$. The excitation of the non-steady-state photo-EMF is carried out in a semiinsulating GaAs crystal. The sample has dimensions of $3 \times 3 \times 0.5 \mathrm{~mm}^{3}$, the front and back surfaces $3 \times 3 \mathrm{~mm}^{2}$ were polished. Two gold stripes were deposited on the front surface (with an interelectrode spacing of $1 \mathrm{~mm}$ ). The sample is tilted around the direction of light propagation by $\sim 15^{\circ}$ in order to decrease contact the photo-EMF [14]. The non-steady-state photo-EMF creates voltage on the load resistor $R_{L}=1 \mathrm{M} \Omega$, which is then measured by the digital oscilloscope. The sweep voltage from the spectrum analyzer feeds the oscilloscope as well, allowing simultaneous measurements of the frequency shift and investigated signal.

Experimental results. - First let us find out if the moment of the signal appearance is unambiguously determined by the slowing-down and stopping of the interference pattern, i.e. by the coincidence of frequencies of the signal and reference beams. We settle several different central frequencies on the spectrum analyzer $(79.6-80.4 \mathrm{MHz})$ and measure the corresponding traces of the non-steadystate photo-EMF (fig. 2). As seen, the pulse position, i.e. the time when photocurrent changes its sign, well corresponds to the moments when the frequencies of the light waves are equal $(\Delta f=0)$.

Figure 3 presents traces of the non-steady-state photoEMF signal measured at different sweep rates $A$. At low sweep rates the pulse consists of two parts with comparable amplitudes and opposite signs. The increase of the sweep rate decreases the pulse duration, while the amplitude remains nearly constant. At large sweep rates the negative peak becomes sharper than the positive one. At

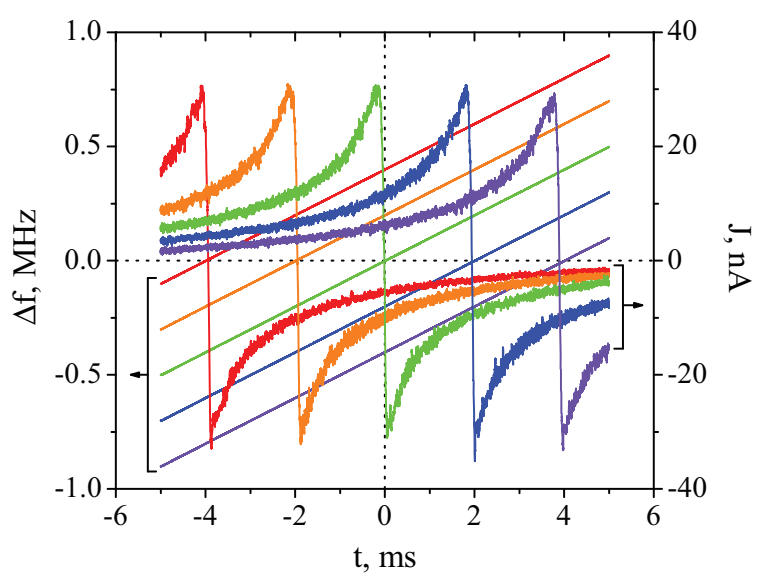

Fig. 2: (Colour on-line) Oscillograms of the frequency shift in the signal beam and the corresponding current arising in a GaAs crystal $\left(A=10^{8} \mathrm{~Hz} / \mathrm{s}\right)$.

very large $A$ chirped and damped oscillations appear. The most pronounced oscillations are achieved by the simultaneous increase of the sweep rate and decrease of the illumination level. The appearance of oscillations can be simply explained. The interference pattern runs very fast at $t<0$ preventing the formation of a space-charge field grating. Then it slows down and stops at $t=0$. The stationary field grating with a noticeable amplitude arises. At $0<t<\tau_{s c}$ the interference pattern begins to run in the opposite direction but the recorded stationary grating is still present. This combination of running interference pattern and stationary grating gives rise to the oscillations of the current analogous to the ones in experiments with conventional non-steady-state photo-EMF. These oscillations are decaying since the space-charge field grating erases with characteristic time $\tau_{s c}[15,16]$.

Let us estimate the pulse response for the case, when frequency deviation $\Delta f(t)=A t$ is very slow. Then, we can consider the non-steady-state photo-EMF as if it is excited by an interference pattern running with constant speed. Here we should clarify the criterion of the mentioned "slowness". Let us compare the time-dependent component of the interference pattern traveling with constant speed with that of the uniformly accelerated pattern. In both cases it can be presented as $\exp [i K x+i \varphi(t)]$, where $\varphi(t)$ is the phase of the interference pattern, which possess the value $\varphi(t)=-2 \pi \Delta f_{0} t$ or $\varphi(t)=-2 \pi \int_{0}^{t} \Delta f(t) \mathrm{d} t=$ $-\pi A t^{2}$, respectively. If we follow the evolution of these phases in the vicinity of time $t_{0}$ during time interval $\Delta t$ (track wave trains of duration $\Delta t$ ), we obtain the corresponding increments:

$$
\varphi\left(t_{0}+\Delta t\right)-\varphi\left(t_{0}\right)=\left\{\begin{array}{l}
-2 \pi \Delta f_{0} \Delta t \\
-2 \pi \Delta f\left(t_{0}\right) \Delta t-\pi A(\Delta t)^{2} .
\end{array}\right.
$$

If the initial frequency shifts at time $t_{0}$ are equal $\left(\Delta f_{0}=\Delta f\left(t_{0}\right)\right)$, then the difference between the considered cases is defined by the term $\pi A(\Delta t)^{2}$. Now we should choose the appropriate value for the time interval $\Delta t$. The 

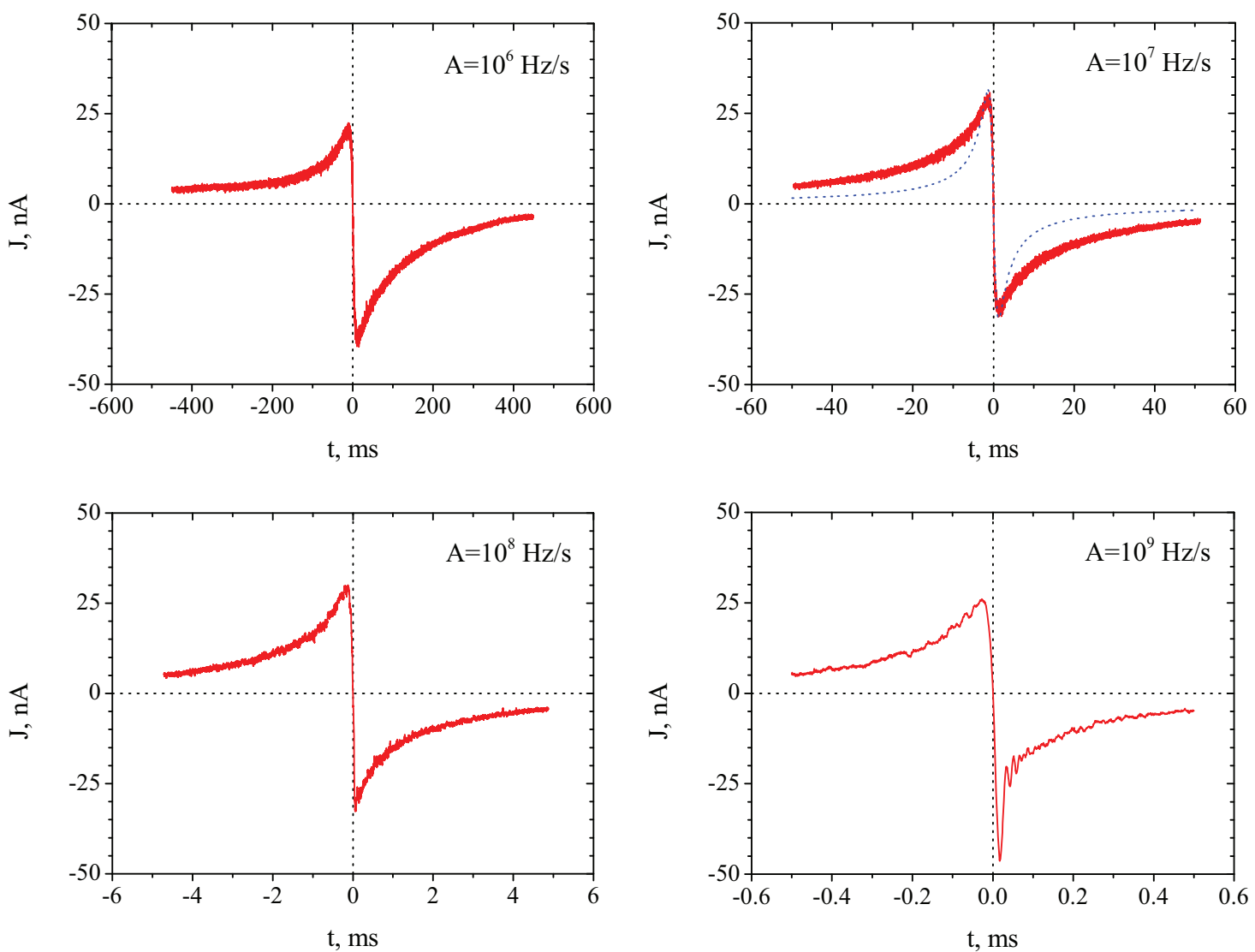

Fig. 3: (Colour on-line) Oscillograms of the photocurrent in a GaAs crystal measured for different sweep rates $A=10^{6}, 10^{7}$, $10^{8}, 10^{9} \mathrm{~Hz} / \mathrm{s}$. The dotted line shows the approximation by eq. (4) for $\tau_{s c}=12 \mu \mathrm{s}$.

time of the space-charge grating formation $\tau_{s c}$ is the best candidate for this role: this is a characteristic amount of time while our dynamic system "remembers" its initial state; in other words, any differences in recording process are insignificant beyond this time interval. Finally, the required criterion is as follows:

$$
\pi A \tau_{s c}^{2} \ll 1
$$

The density of the photocurrent excited by the interference pattern running with constant speed [10] is given by

$$
j=\frac{m^{2} \sigma_{0} E_{D}}{2\left(1+K^{2} L_{D}^{2}\right)} \frac{\Delta \omega \tau_{s c}}{1+\left(\Delta \omega \tau_{s c}\right)^{2}},
$$

where $\sigma_{0}$ is the average photoconductivity, $E_{D}$ is the diffusion field, $L_{D}$ is the diffusion length of the photocarriers. Here we assume the spatial frequency to be not very high: $K^{2} L_{S}^{2} \ll 1$, where $L_{S}$ is the Debye screening length. The direct substitution of the frequency shift $\Delta \omega(t)=2 \pi \Delta f(t)=2 \pi A t$ provides the desired pulse response:

$$
j(t)=\frac{m^{2} \sigma_{0} E_{D}}{2\left(1+K^{2} L_{D}^{2}\right)} \frac{2 \pi A \tau_{s c} t}{1+\left(2 \pi A \tau_{s c} t\right)^{2}} .
$$

According to this expression the pulse should appear in the vicinity of $t=0$ where the interference pattern is stopped. It should consist of the negative and positive parts, the peak-to-peak amplitude $m^{2} \sigma_{0} E_{D} / 2\left(1+K^{2} L_{D}^{2}\right)$ is determined generally by the average photoconductivity. The duration of the pulse $\tau_{p} \simeq 1.19 / A \tau_{s c}$ defined by the relative level \pm 0.5 depends on the sweep rate $A$ (condition of the experiment) and time $\tau_{s c}$ (parameter of the material).

As seen from this expression, the main features of the effect such as the time of the pulse appearance, the shape of the pulse and the variation of its duration vs. sweep rate are well matched to the experiment. There are, however, some peculiarities which are not described by eq. (4). First, the pulse measured at the lowest sweep rate ( $\left.A=10^{6} \mathrm{~Hz} / \mathrm{s}\right)$ should be the most symmetric. In practice the preceding positive half has a 1.9 times smaller amplitude than the following negative one. Second, the pulse tails decay more slowly than $t^{-1}$ as predicted by eq. (4). The dotted line in fig. 3 shows the approximation by eq. (4) for $\tau_{s c}=12 \mu \mathrm{s}$ (we fitted the most symmetric curve). The origin of these discrepancies is unknown, we just can suppose that it can be associated with bipolar conductivity and high light absorption in GaAs at $\lambda=633 \mathrm{~nm}$. Both factors sufficiently change the dynamics of space-charge formation and non-steady-state photo-EMF characteristics [17-19]. 


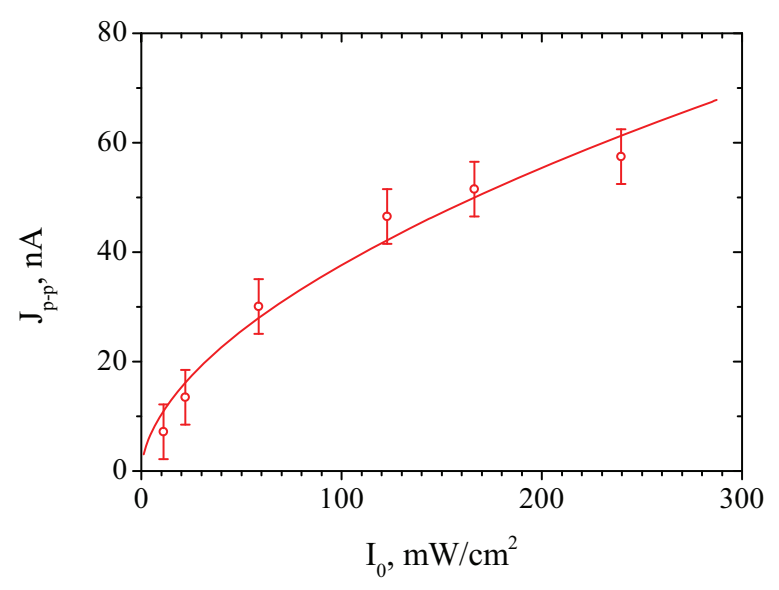

Fig. 4: (Colour on-line) Dependence of the photo-EMF peakto-peak amplitude vs. average light intensity $(A=5 \times$ $\left.10^{7} \mathrm{~Hz} / \mathrm{s}\right)$. The solid line shows the approximation $J_{p-p} \propto I_{0}^{0.56}$.

We have also measured the dependence of the signal on the light intensity (fig. 4). The peak-to-peak amplitude of the pulse demonstrates noticeable sublinearity $J_{p-p} \propto I_{0}^{0.56}$ in the range $I_{0}=11-240 \mathrm{~mW} / \mathrm{cm}^{2}$, which is rather typical for GaAs crystal at this wavelength. Decreasing the light intensity we observed an evolution of the pulse shape similar to that for the increasing sweep rate. This fact confirms the statement that the pulse shape is determined by the combination of the parameters $A$ and $\tau_{s c}$.

Discussion. - The uniformly accelerated interference pattern excites the pulse of electric current in semiconductors. For a slowly varying frequency shift $\left(\pi A \tau_{s c}^{2} \ll 1\right)$ we can use a theoretical approach developed earlier for the interference pattern running with constant speed [10]. However, some features such as oscillations arising at higher sweep rates $\left(\pi A \tau_{s c}^{2} \geq 1\right)$ and slowly decaying pulse tails in GaAs point out to the necessity of a more detailed theoretical analysis taking into account the dynamics of space-charge formation, bipolar conductivity, high light absorption [17-19].

For years we emphasized the fact that the frequency transfer function of the conventional non-steady-state photo-EMF measured at low frequencies is similar to that of the simplest differentiating RC-circuit (high-pass filter) $[2,3]$. One can note that the behavior of the photo-EMF signal for linear frequency modulation is more similar to the one of the integrating RC-circuit (low-pass filter): at large $|t|$ the frequency is large, too, and the ac voltage is damped; at low $|t|$, when the frequency is small, the signal freely passes through the integrating RC-circuit.

Let us point out the possible applications of the studied effect. The pulses appear at the moments when the frequencies of the signal and reference beams coincide. This fact can be used in the Doppler scanning velocimeter estimating the unknown velocity from the time delay of the detected pulses. Moreover, the duration and shape of the pulses provide information about the sweep rate of the
LFM signal, which can be caused by the uniformly accelerated motion of the object. This means that the velocity and acceleration can be measured simultaneously by the detection of the only pulse, which is very actual in investigations of fast processes. In this work we used frequency shifts and sweep rates in the ranges $|\Delta f|=0-10 \mathrm{MHz}$ and $|A|=1-1000 \mathrm{MHz} / \mathrm{s}$. They would correspond to the following ranges of velocity and acceleration of the light reflecting object: $|V|=0-3 \mathrm{~m} / \mathrm{s}$ and $|a|=0.6-600 \mathrm{~m} / \mathrm{s}^{2}$.

The devices realized on the considered effect will inherit the advantages of the devices using conventional nonsteady-state photo-EMF. They include the rather high sensitivity and adaptivity, i.e. the possibility of operation in the presence of a slow phase drift in the interferometric setup. The devices will also be able to detect light signals with complicated wave fronts, even with speckle patterns produced by reflection from the real diffusely scattering objects.

The developed techniques can also be used for characterization of wide-gap semiconductors. Instead of investigations of frequency transfer functions we can measure the pulse response and estimate space-charge formation time $\tau_{s c}$ and the corresponding photoconductivity $\sigma_{0}$.

Conclusion. - Linear frequency modulation of optical beams provides another way to excite time-dependent photo-EMF signal. The effect reveals itself as an electric pulse arising in photoconductive media at the moments when the interference pattern slows down and stops. Duration, amplitude and shape of the pulses are determined by the sweep rate of linear frequency modulation and by the material parameters. This may allow to develop new techniques of material characterization and the detection of frequency-modulated optical signals.

MB, VK, and IS acknowledge the financial support from the Ministry of Knowledge Economy of Korea.

\section{REFERENCES}

[1] Trofimov G. and Stepanov S., Sov. Phys. Solid State, 28 (1986) 1559.

[2] Petrov M., Sokolov I., Stepanov S. and Trofimov G., J. Appl. Phys., 68 (1990) 2216.

[3] Sokolov I. and Stepanov S., J. Opt. Soc. Am. B, 10 (1993) 1483.

[4] Kukhtarev N., Kukhtareva T., Lyuksyutov S., Reagan M., Banerjee P. and Buchhave P., J. Opt. Soc. Am. B, 22 (2005) 1917.

[5] Haken U., Hundhausen M. and Ley L., Phys. Rev. B, 51 (1995) 10579.

[6] Veenhuis H., Buse K., Krätzig E., Korneev N. and Mayorga D., J. Appl. Phys., 86 (1999) 2389.

[7] Gather M., Mansurova S. and Meerholz K., Phys. Rev. B, 75 (2007) 165203. 
[8] dos Santos T., Frejlich J. and Shcherbin K., Appl. Phys. B, 99 (2010) 701.

[9] Stepanov S., Sokolov I., Trofimov G., Vlad V., Popa D. and Apostol I., Opt. Lett., 15 (1990) 1239.

[10] Wang C., Davidson F. and Trivedi S., Appl. Opt., 34 (1995) 6496.

[11] Pepper D., Dunning G., Nolte D., Coy J., Pouet B., Bacher G. and Klein M., Opt. Photon. News, 10 (1999) 11.

[12] dos Santos T., Frejlich J., Launay J. and Shcherbin K., Appl. Phys. B , 95 (2009) 627632.

[13] Mansurova S., Moreno Zarate P., Rodriguez P., Stepanov S., Köber S. and Meerholz K., Opt. Lett., 37 (2012) 383.
[14] Trofimov G., Stepanov S., Petrov M. and Krasin'Kova M., Sov. Tech. Phys. Lett., 13 (1987) 108.

[15] Günter P. and Huignard J.-P., Photorefractive Materials and their Applications (Springer-Verlag, Berlin) 1989.

[16] Petrov M., Stepanov S. and Khomenko A., Photorefractive Crystals in Coherent Optical Systems (Springer-Verlag, Berlin) 1991.

[17] Stepanov S. and Trofimov G., Sov. Phys. Solid State, 31 (1989) 49.

[18] Korneev N. and Stepanov S., J. Appl. Phys., 74 (1993) 2736.

[19] Korneev N., Mansurova S. and Stepanov S., J. Appl. Phys., 78 (1995) 2925. 